\title{
Structural Characterization of Histone H2A Variants
}

\author{
S. Chakravarthy ${ }^{*}$ Y. Bao, ${ }^{*}$ V.A. Roberts, ${ }^{\dagger}$ D. Tremethick, ${ }^{\dagger}$ And K. Luger ${ }^{*}$ \\ *Department of Biochemistry and Molecular Biology, Colorado State University, Fort Collins, \\ Colorado 80523-1870; 'Department of Molecular Biology, The Scripps Research Institute, La Jolla, \\ California 92037; The John Curtin School of Medical Research, The Australian National University, \\ Australian Capital University, Canberra ACT, 2601 Australia
}

Eukaryotic DNA associates with an equal amount of protein to form chromatin, the fundamental unit of which is the nucleosome core particle (NCP). An NCP consists of two copies each of the four core histones H2A, H2B, $\mathrm{H} 3$, and H4. This histone octamer binds 147 base pairs of DNA around its outer surface in 1.65 tight superhelical turns (Fig. 1A) (Luger et al. 1997; Richmond and Davey 2003). Linker histones and other nonhistone proteins promote or stabilize the folding of nucleosomal arrays into superstructures of increasing complexity and largely unknown architecture (Hansen 2002). Covalent modification of the core histones and variations in the fundamental biochemical composition of nucleosomes distinguish transcriptionally active from inactive chromatin regions, by either changing the structure of the nucleosomes, altering their ability to interact with other protein factors, or modifying their propensity to fold into varying degrees of higher-order structures (or by any combination of the above). Studying the mechanism for establishing distinct chromatin domains is essential to understanding differential regulation of gene expression and all other DNA-de- pendent processes. Much progress has been made in this direction in the past few years.

Substitution of one or more of the core histones with the corresponding histone variants has the potential to exert considerable influence on the structure and function of chromatin. Histone variants are distinct nonallelic forms of conventional, major-type histones that form the bulk of nucleosomes during replication and whose synthesis is tightly coupled to $\mathrm{S}$ phase. Histone variants are characterized by a completely different expression pattern that is not restricted to $\mathrm{S}$ phase. They are found in most eukaryotic organisms and are expressed in all tissue types (unlike some $\mathrm{H} 2 \mathrm{~B}$ isoforms that are found only in specialized tissues such as testes). Compared to their majortype counterparts, histone variants exhibit moderate to significant degrees of sequence homology (Fig. 1B). H2A.X $(82 \%)$ and H3.3 ( 96\%) are the least divergent of all histone variants. H2A.Z ( $60 \%)$, macroH2A $(\sim 65 \%)$, H2A.Bbd (40\%), and CenpA, which has a 93 amino acid domain that is $62 \%$ identical to H3 (Palmer et al. 1991; Sullivan et al. 1994), are increasingly divergent in their

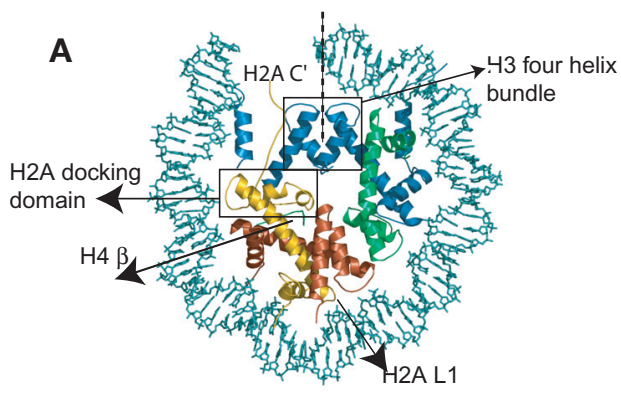

Figure 1. Histone H2A variants. (A) Overview of nucleosome structure. Only 74 base pairs of DNA and associated proteins are shown. Yellow: H2A; red: H2B; blue: H3; green: H4. The axis of noncrystallographic symmetry is indicated by a dashed line. The four-helix bundle structure formed by the two $\mathrm{H} 3$ molecules and the H2A docking domain are boxed; other structural features are indicated. $(B)$ Sequence alignment of the histone domain of human H2A.X, mouse H2A.Z, mouse macroH2A, and human H2A.Bbd with majortype mouse H2A. Bullets: every tenth residue in major H2A; black: identical residues; blue: similar residues; red: different residues. Also indicated are the secondary structure elements of the histone fold $(\alpha 1, \alpha 2$, and $\alpha 3)$ and the loops and extensions (L1, L2, $\alpha \mathrm{N}$, and $\alpha \mathrm{C})$.
B

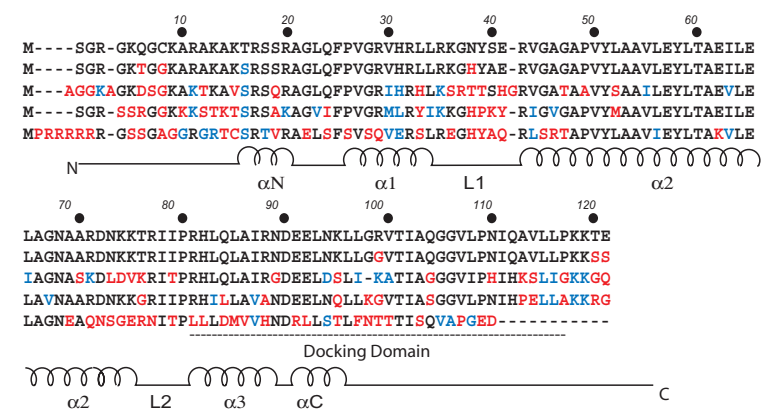
M- - --SGR-GKTGGKARAKAKSRSSRAGLOFPVGRVHRLLRKGHYAE-RVGAGAPVYLAAVLEYLTAEILE M M- -- SGR-SSRGGKKKSTKTSRSAKAGVIFPVGRMLRYIKKGHPKY-RIGVGAPVYMAAVLEYLTAEILE MPRRRRRR-GSSGAGGRGRTCSRTVRAELSFSVSOVERSLREGHYAO-RLSRTAPVYLAAVIEYLTAKVLE 
histone moiety from $\mathrm{H} 2 \mathrm{~A}$ and $\mathrm{H} 3$, respectively. As is the case with histones in general, the structured regions of the histones (encompassing histone folds and extensions) are more conserved than the histone tails. The structured region of $\mathrm{H} 2 \mathrm{~A}$.X is $97 \%$ conserved to its major-type $\mathrm{H} 2 \mathrm{~A}$ counterpart, that of macroH $2 \mathrm{~A}, 70 \%$, of $\mathrm{H} 2 \mathrm{~A} . \mathrm{Z}, 66 \%$, and of $\mathrm{H} 2 \mathrm{~A} . \mathrm{Bbd}, 48 \%$. MacroH2A is unique in that it contains an additional nonhistone-like domain that is connected to the histone-homology domain by a flexible linker (Pehrson and Fried 1992). All histone variants are highly conserved between different species. In many cases, they are even more conserved than their majortype paralogs (Sullivan et al. 2002), indicating that they all have evolved to fulfill important functions that cannot be accomplished by major-type $\mathrm{H} 2 \mathrm{~A}$ and $\mathrm{H} 3$, as has been demonstrated for H2A.Z (van Daal and Elgin 1992; Clarkson et al. 1999; Faast et al. 2001).

While the modus operandi of most of histone variants remains unknown, they are all characterized by unique in vivo localization patterns, which in turn shed light on their putative function. H2A.X is distributed throughout the genome. It is implicated in double-stranded DNA repair (Celeste et al. 2003; Rothkamm and Lobrich 2003) and is necessary for programming DNA breakage that occurs in developing lymphocytes (Bassing et al. 2003). The presence of H2A.Z within euchromatin plays a role in preventing silencing from spreading into regions of the chromosome that are normally transcriptionally active (Meneghini et al. 2003). Interestingly, H2A.Z can coexist with Sir proteins at the telomere (Krogan et al. 2003). Most recently, H2A.Z has also been shown to play a role in chromosome segregation (Rangasamy et al. 2004). $\mathrm{MacroH} 2 \mathrm{~A}$ is found at the inactive $\mathrm{X}$ chromosome of adult female mammals, which consists predominantly of heterochromatin and is transcriptionally inactive (Costanzi and Pehrson 1998), while H2A.Bbd colocalizes with hyperacetylated histone $\mathrm{H} 4$, indicating that it might be associated with actively transcribed chromatin (Chadwick and Willard 2001). The histone H3 variant H3.3 is thought to be associated with active chromatin, and CENP-A is a major component of centromeric heterochromatin (Vermaak and Wolffe 1998; Ahmad and Henikoff 2002a,b).

Here we will summarize and review available structural information on nucleosomes and chromatin containing histone H2A variants, and will attempt to explain how structure relates to their varied function. We note that an exhaustive review of all biological and functional data would clearly exceed the scope of this manuscript. We will present a hypothesis why "true" histone variants have been identified for only histone $\mathrm{H} 2 \mathrm{~A}$ and $\mathrm{H} 3$, and we will show data in support of our hypothesis that particular regions in the $\mathrm{H} 2 \mathrm{~A}$ amino acid sequence appeared to have been targets during the evolution of $\mathrm{H} 2 \mathrm{~A}$ histone variants.

\section{WHY ARE THERE NO H4 AND H2B HISTONE VARIANTS?}

All known true histone variants are replacements for either histone $\mathrm{H} 3$ or $\mathrm{H} 2 \mathrm{~A}$ (for review, see Malik and Henikoff 2003; Henikoff et al. 2004). From a structural vantage point, we hypothesize that this is the case because only $\mathrm{H} 3$ and $\mathrm{H} 2 \mathrm{~A}$ are engaged in homotypic interactions (Fig. 2). In contrast, neither $\mathrm{H} 4$ nor H2B interact with the other $\mathrm{H} 4$ or $\mathrm{H} 2 \mathrm{~B}$ molecule within the histone octamer. A four-helix bundle formed by residues from the two H3 chains holds together the $(\mathrm{H} 3-\mathrm{H} 4)_{2}$ tetramer, which is stable in the absence of DNA under physiological conditions (Fig. 2A,B). This interface, which is characterized by a combination of salt bridges, ionic interactions, and some hydrophobic contacts (Luger et al. 1997), had been proposed many years ago to be conformationally flexible, especially in the absence of the (H2A-H2B) dimers (Chen et al. 1991; Hamiche et al. 1996; but see also Protacio and Widom 1996). In contrast, the interface formed between two $\mathrm{H} 2 \mathrm{~A}$ molecules is quite small and only exists in the context of a folded nucleosome (Fig. $2 \mathrm{C}, \mathrm{D})$. It is, however, the only point of contact between the two (H2A-H2B) dimers in a nucleosome, and thus may be responsible in part for highly cooperative incorporation of the two H2A-H2B dimers, as well as for tethering the two gyres of the DNA superhelix together. Intriguingly, major sequence differences between $\mathrm{H} 3$ and the centromeric H3 variant are found in this four-helix bundle region (Shelby et al. 1997; Black et al. 2004). Similarly, variability among the many H2A variants themselves and differences between variants and majortype H2A are found in the L1 loop (Fig. 1B). This sug-
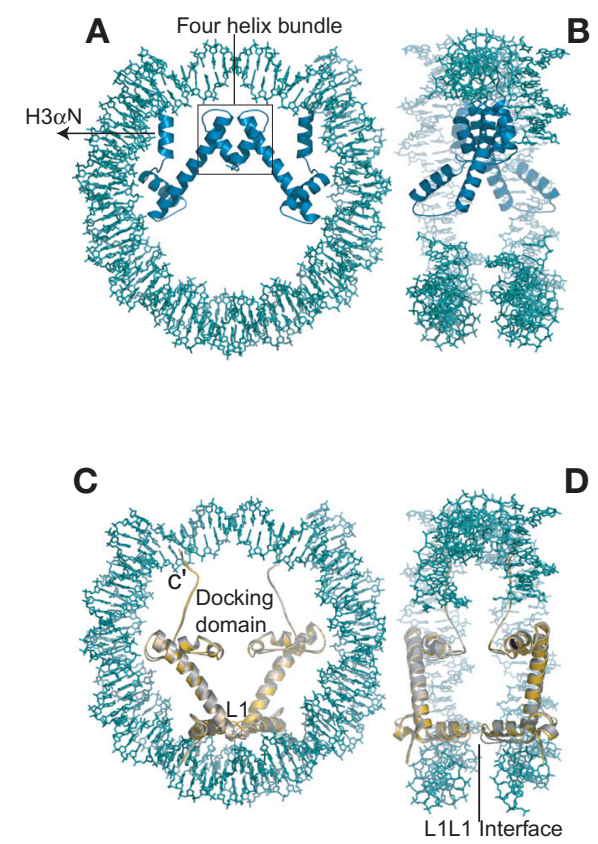

Figure 2. $\mathrm{H} 3$ and $\mathrm{H} 2 \mathrm{~A}$ fulfill special roles within the NCP. ( $A$ ) Structure of the NCP viewed down the superhelical axis. Only the two H3 chains are shown. $(B)$ The same structure is shown after rotation by $90^{\circ}$ around the $y$-axis, with parts of the DNA omitted for clarity. (C) Structure of the NCP viewed down the superhelical axis. Only the two H2A chains are shown. The structures of NCPs containing major-type H2A from Xenopus laevis (yellow; pdb entry code 1AOI), H2A.Z (wheat; pdb entry code 1F66), and macro-H2A (gray; S. Chakravarthy, unpubl.) have been superimposed. $(D)$ The structures displayed in $C$, shown in the same orientation as $B$. 
gests that sequence variability in these regions of self-interactions may serve to ensure that only nucleosomes with two identical H2A or H3 "flavors" are formed. Equally likely, these interfaces may preclude the formation of such nucleosomes and may instead favor the incorporation of a major-type histone $\mathrm{H} 3$ with variant $\mathrm{H} 3$, or major-type $\mathrm{H} 2 \mathrm{~A}$ with variant $\mathrm{H} 2 \mathrm{~A}$, respectively, as will be shown below.

A second common feature of $\mathrm{H} 3$ and $\mathrm{H} 2 \mathrm{~A}$ not shared by $\mathrm{H} 4$ and $\mathrm{H} 2 \mathrm{~B}$ is the fact that they both are involved in the organization of more than the requisite $\sim 70$ base pairs of DNA that are bound by a canonical histone fold dimer (Luger and Richmond 1998a), because of quite extended regions in both $\mathrm{H} 3$ and $\mathrm{H} 2 \mathrm{~A}$ outside the histone fold. The amino-terminal helix of $\mathrm{H} 3(\alpha \mathrm{N})$ is positioned to interact with the penultimate turn of the DNA double helix before it exits the confines of the nucleosome (Figs. 1A and 2A). It is held in position by the $\mathrm{H} 2 \mathrm{~A}$ docking domain (Bao et al. 2004). The carboxy-terminal tail of $\mathrm{H} 2 \mathrm{~A}$ is poised to interact with linker DNA that extends beyond the $147 \mathrm{nu}-$ cleosomal base pairs (Figs. 1A and 2C).

Thus, unlike $\mathrm{H} 2 \mathrm{~B}$ and $\mathrm{H} 4$, replacement of $\mathrm{H} 3$ and $\mathrm{H} 2 \mathrm{~A}$ with histone variants has the potential to affect DNA organization (or exit angle) at the penultimate 15 base pairs of nucleosomal DNA, with potential implications for higher-order structure. As pointed out above, sequence variations that are specific to either $\mathrm{H} 3$ or $\mathrm{H} 2 \mathrm{~A}$ variants have the potential to increase the pool of theoretically possible nucleosomes, in being able to choose their interaction partners. Finally, in light of the current models for linker histone binding to the nucleosome (Crane-Robinson 1997; Hayes and Hansen 2001), histone H1 and its variants and isoforms are more likely to interact with $\mathrm{H} 3$ and $\mathrm{H} 2 \mathrm{~A}$ than with $\mathrm{H} 2 \mathrm{~B}$ and $\mathrm{H} 4$. One intriguing (but as yet untested) model for how histone variants exert their function in chromatin may be by modulating the interaction between the nucleosome and linker histones or nonhistone architectural chromatin proteins, such as HP1. For example, it is conceivable that nucleosomes harboring certain $\mathrm{H} 2 \mathrm{~A}$ or $\mathrm{H} 3$ variants may be unable to interact with linker histones or other architectural proteins, or that they may prefer certain $\mathrm{H} 1$ isoforms over others, with profound effects on gene regulation and/or chromatin higher-order structure.

\section{STRUCTURAL CHARACTERISTICS OF NUCLEOSOMES AND CHROMATIN CONTAINING HISTONE H2A VARIANTS}

\section{H2A.X}

Amino acids (1-120) of H2A.X are very similar in sequence to major $\mathrm{H} 2 \mathrm{~A}$; indeed, with an only two amino acid difference in the structured domain it is safe to assume that the structural properties of a mononucleosome are likely to remain unaffected. The two sequence changes in the structured domain of H2A.X are in the L1 loop and in the docking domain, respectively, and are also found to distinguish macroH2A from major-type H2A (H2A N38 to H, and R99 to G, Fig. 1B).

The carboxy-terminal domain of H2A.X is phosphory- lated, and it is this phosphorylated form of this variant that is implicated in DNA repair (Celeste et al. 2003). One possible mode of H2A.X action could be via recruitment of repair proteins to the site of DNA damage. It is indeed found that formation of Nbs1, 53bp1, and Brcal foci at the damage sites is severely impaired in H2A.X-/cells, as well as suppressing genomic instability (Celeste et al. 2003).

\section{H2A.Z}

H2A.Z, which is essential in Drosophila, mouse, and Tetrahymena (van Daal and Elgin 1992; Liu et al. 1996; Faast et al. 2001), is the histone variant whose structure and function is perhaps best studied among all histone variants. While not being essential in budding yeast, H2A.Z (Htz1) functions to prevent the spread of heterochromatin into euchromatin (Meneghini et al. 2003) and plays a role in transcription with a function that is partially redundant with specific chromatin remodeling complexes.

The crystal structure of an NCP in which major H2A is replaced by H2A.Z reveals no major differences in the path of the DNA superhelix or in the nature of protein-DNA interactions (Suto et al. 2000). While tyrosine quenching revealed no major change in nucleosome stability in vitro, a more sensitive fluorescence resonance energy transfer (FRET) approach (Park et al. 2004) revealed that the sequence changes of H2A.Z result in subtle stabilization of the (H2A-H2B) dimer interaction with the $(\mathrm{H} 3-\mathrm{H} 4)_{2}$ tetramer-DNA complex upon increasing ionic strength (Fig. 3).

Amino acid changes in the H2A.Z docking domain also contribute to an extended acidic patch, which is a prominent feature of the nucleosome surface (Fig. 4) (Suto et al. 2003). The docking domain is the region that

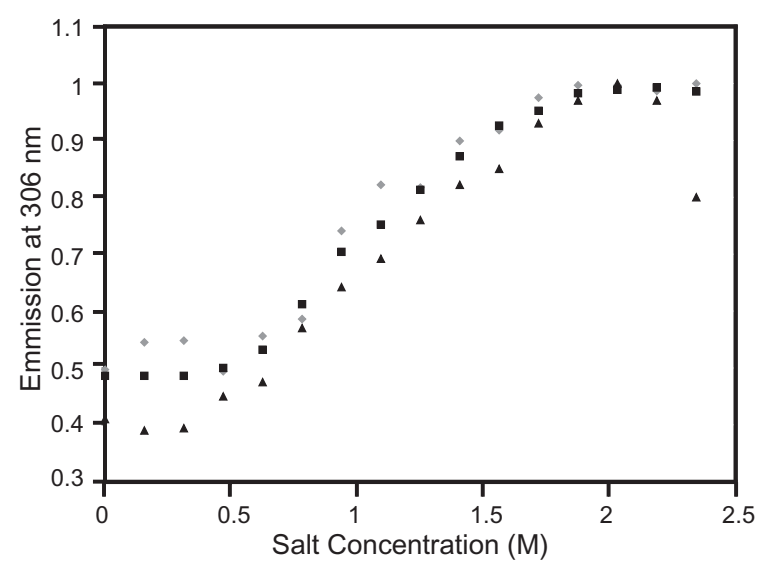

Figure 3. The tyrosine fluorescence quenching profile shows no pronounced differences in the overall stability of macro-NCP, H2A.Z-NCP, and major-NCP. Tyrosine quenching profiles in response to increased ionic strength for nucleosomes in which the histone domain of macroH2A, or H2A.Z, has replaced major $\mathrm{H} 2 \mathrm{~A}$. Values have been normalized using the formula $A_{n}=A / A_{h}$, where $A_{n}$ is the normalized value, $A$ is the observed signal, and $A_{h}$ is the highest signal observed. Diamonds: major-NCP; squares: macro-NCP; triangles: H2A.Z-NCP. 
is essential for Drosophila development (Clarkson et al. 1999). This region of H2A.Z (and the amino-terminal tail) has been shown to interact with the pericentric heterochromatin-binding protein INCENP (Rangasamy et al. 2003), a protein critical for proper chromosome segregation. Thus, it appears that subtle sequence differences between major-type H2A and H2A.Z bear a direct relevance to its ability to interact with nonhistone proteins.

Restriction digest analysis of an in vitro chromatin model system (H2A or H2A.Z nucleosome arrays assembled on a DNA template that contains 12 repeats of a 208base pair nucleosome positioning sequence) in combination with sedimentation velocity studies established that H2A and H2A.Z nucleosomes assemble with comparable affinity (Fan et al. 2002). Consistent with the increase in nucleosome core stability, H2A.Z arrays are more regularly spaced than $\mathrm{H} 2 \mathrm{~A}$ arrays. Sedimentation velocity analysis at elevated salt concentrations was used to determine that H2A.Z facilitates intramolecular folding of nucleosome arrays resulting in the formation of a folded state (55S). This folded state is thought to reflect the canonical $30-\mathrm{nm}$ fiber. On the other hand, H2A.Z is found to hinder the oligomerization mediated by interactions between nucleosome arrays (Fan et al. 2002). Therefore, H2A.Z arrays form higher-order chromatin structures distinctly different from $\mathrm{H} 2 \mathrm{~A}$ arrays. Consistent with this, H2A.Z is located at constitutive heterochromatin in mammalian cells and is required for faithful chromosome segregation (Rangasamy et al. 2003, 2004). Taken together, H2A.Z may have a dual role in modulating the recruitment of nonhistone proteins and in chromatin fiber folding. These features would enable H2A.Z to play a role in a number of different processes that include gene expression and mitosis.

\section{$\operatorname{macroH} 2 \mathrm{~A}$}

macroH2A is perhaps the most unusual of all histone variants because of its large size ( $\sim 370$ amino acids and a molecular weight of $42 \mathrm{kD}$ ) and tripartite structural organization. The amino-terminal third (amino acid 1-122) is $\sim 64 \%$ identical to major H2A (Pehrson and Fried 1992). This region is followed by a highly basic stretch (amino acid 132-160), which is homologous to the carboxyl terminus of linker histone H1 (57\% identical to sea urchin H1 $\gamma$ over 30 residues; Pehrson and Fried 1992). Amino acids $161-370$ form a tightly folded domain whose highresolution structure we have determined recently (G.S.Y.K. Swamy and S. Chakravarthy, unpubl.). The structure of an NCP in which $\mathrm{H} 2 \mathrm{~A}$ has been replaced by the histone domain of macroH2A shows that the significant differences in sequence are accommodated with surprisingly minor structural changes (Fig. 2C,D) (S. Chakravarthy et al., unpubl.). The most pronounced structural divergence is found in the L1 region of macroH2A. Changes in the electrophoretic behavior of mononucleosomes containing the H2A-like domain of macroH2A, in the stability of the histone octamer at decreased ionic strength, and in the absence of the stabilizing influence of the DNA were observed. A mutant of major-type H2A in which four amino acids in the L1 loop of major-type H2A (38-NYAE-41) were replaced with the corresponding amino acids from macroH2A (38-HPKY-41) was shown to confer all in vitro characteristics of macroH2A-containing octamer and $\mathrm{NCP}$ onto major-type H2A (S. Chakravarthy et al., unpubl.).

Despite the pronounced effect on histone octamer stability, preliminary data show that the overall stability of the nucleosome in response to increased ionic strength is not affected (Fig. 3). However, given our results with H2A.Z-containing nucleosomes (Park et al. 2004), it is possible and even likely that more subtle changes exist. It should be pointed out that since the first and foremost task of histone variants is to form nucleosomes, subtle changes are expected at most, but clearly these differences are fundamental to the function of histone variants.

macroH2A-containing chromatin has been shown to be inhibitory to transcription in vivo (Perche et al. 2000). Furthermore, macroH2A-containing mononucleosomes are not remodeled by SWI/SNF and are unable to bind transcription factors (Angelov et al. 2003). More precisely, the nonhistone domain or the flexible linker (or both) were responsible for the inability of these variant nucleosomes to bind the transcription factor NF- $\mathrm{kB}$. In contrast, the histone-like domain of macroH2A was shown to be responsible for the inability of macroH2Acontaining nucleosomes to be remodeled (Angelov et al. 2003). It will be of interest to see whether the L1 loop alone can confer this inhibition of chromatin remodeling and transcription to nucleosomes. The key location of the L1 loop within the NCP structure (where it seemingly holds together the two gyres of the DNA double helix) certainly makes this region a prime candidate to affect the dynamic motions of the NCP associated with chromatin remodeling.

macroH2A displays a very unique nuclear localization pattern. While it is expressed in equal quantities in both males and females (Rasmussen et al. 1999), in almost all the higher eukaryotes it is enriched in the inactive $\mathrm{X}$ chromosome (Xi) of adult female mammals manifested as a Macro Chromatin Body (MCB) (Costanzi and Pehrson 1998; Hoyer-Fender et al. 2000). Various GFP-tagged constructs have been used to determine the importance of the histone and the nonhistone domain in the localization of macroH2A to the inactive $\mathrm{X}$ chromosome (Chadwick et al. 2001). The histone domain alone (without aminoand carboxy-terminal tails) fused to GFP was found to form the Xi-associated MCB with efficiencies comparable to full-length macroH2A. Of the 19 amino acids that are different in macroH2A compared to major $\mathrm{H} 2 \mathrm{~A}$, no single amino acid could be attributed this function by point mutation studies. On the basis of preliminary analysis of the crystal structure of macroH2A mononucleosomes and biochemical studies of L1 mutants of major H2A, it seemed likely that the L1 loop is crucial for the localization on the Xi. This hypothesis has been tested experimentally (S. Chakravarthy et al., unpubl.).

macroH2A is gradually emerging as a "family" of variants. At least two different genes are known to encode this protein with significant variations in sequence but the 
same basic structural organization (H2A1 and macroH2A2, respectively). macroH2A1 has two splice variants (macroH2A1.1 and macroH2A1.2), which are nonidentical in a very small region of the nonhistone region starting at amino acid 195 (Pehrson et al. 1997). macroH2A2 is overall $68 \%$ identical to macroH2A1.2. The histone region is $84 \%$ identical to that of macroH2A1 and only $66 \%$ identical to major $\mathrm{H} 2 \mathrm{~A}$. The sequence of the macroH2A2 L1-loop (TFKY) seems to be a convolution between that of major H2A (NYAE) and macroH2A (HPKY). The basic region is the most varied and is only $25 \%$ identical to that of macroH2 A1, whereas the nonhistone region is $64 \%$ identical to that of macroH2A 1.2 . macroH2A1.2 and macroH2A2 display very similar (and sometimes overlapping) nuclear localization patterns, at least at a global level, and the functional relevance of the sequence differences remains unclear (Chadwick et al. 2001; Costanzi and Pehrson 2001).

\section{H2A.Bbd}

The structured region of H2A.Bbd is only $48 \%$ identical to that of major H2A, making H2A.Bbd the most divergent histone $\mathrm{H} 2 \mathrm{~A}$ variant known to date. So far, this histone variant has been identified in only humans and mice. Major hallmarks of the amino acid sequence of H2A.Bbd as compared to that of major H2A are (1) the presence of a continuous stretch of five arginines and the conspicuous absence of lysines in its amino-terminal tail; (2) the absence of a carboxy-terminal tail and the very last segment of the docking domain; (3) major sequence differences in the docking domain of $\mathrm{H} 2 \mathrm{~A}$; (4) the presence of only one lysine in H2A.Bbd compared to 14 in major $\mathrm{H} 2 \mathrm{~A}$, resulting in a slightly less basic protein (pI 10.7, compared to a pI of 11.2 for major H2A); and (5) the absence of the "acidic patch" (Luger and Richmond 1998b) on the docking domain (Fig. 1B).

We found that mononucleosomes containing H2A.Bbd had a more relaxed structure with less tightly bound DNA ends (Bao et al. 2004). Only $118 \pm 2$ bp of DNA are protected against digestion with micrococcal nuclease, in contrast to $146 \mathrm{bp}$ in canonical nucleosomes. These results are consistent with the observed more rapid exchange of GFP-H2A.Bbd in vivo (Gautier et al. 2004). Intriguingly, we also found a lower repeat length in micrococcal nuclease digestion of nucleosomes reconstituted onto plasmids using a recombinant in vitro assembly system (Georges et al. 2002) (125 bp as opposed to $\sim 160$ bp for major nucleosome arrays), which suggests that the H2A.Bbd nucleosomes are deposited at a higher density. At this high density, H2A.Bbd represses transcription comparable to H2A. Intriguingly, domain swap experiments (in which the H2A docking domain was exchanged with that of Bbd) show that the H2A.Bbd docking domain is largely responsible for its behavior. The conservation of the histone fold together with the nuclear localization pattern suggests that H2A.Bbd alters chromatin structure at the nucleosomal level, giving rise to transcriptionally active domains (Chadwick and Willard 2001).

\section{EVOLUTIONARY TARGETS IN THE HISTONE FOLD OF H2A VARIANTS}

Sequence comparisons (Fig. 1B) together with analysis of the two available crystal structures of nucleosomes containing histone variants (Suto et al. 2000; S. Chakravarthy, unpubl.) and analysis of biochemical and biophysical data from our and several other laboratories show that histone variants are true replacement histones in that they can form functional nucleosomes and chromatin. The majority of structural and functional changes in histone $\mathrm{H} 2 \mathrm{~A}$ variants reside in the docking domain and in the L1 loops, with the latter being more structurally divergent than the former.

\section{The Multifunctional H2A Docking Domain}

The docking domain is involved in interactions between the H2A-H2B dimer and the $(\mathrm{H} 3-\mathrm{H} 4)_{2}$ tetramer and harbors three of the seven residues that form the "acidic patch" on the surface of the nucleosome (Luger and Richmond 1998b). Thus, sequence divergence in this region may affect the stability of the $\mathrm{H} 2 \mathrm{~A}$ variant- $\mathrm{H} 2 \mathrm{~B}$ dimer/(H3-H4) $)_{2}$ tetramer interface, as has been observed for H2A.Z (Park et al. 2004), which will have effects on chromatin remodeling and transcription. The inefficient organization of the penultimate $\sim 15-20$ base pairs of nucleosomal DNA can also be a consequence of relatively minor sequence changes in this domain, as described for H2A.Bbd (Bao et al. 2004). Changes in amino acid sequence may also alter the surface of the nucleosome, with important implications for the ability of nucleosomes to interact with other factors or to form more compact higher-order structures. It is interesting to note that while the acidic patch is decreased in H2A.Bbd (not shown), its size is actually increased in H2A.Z (Suto et al. 2000), and expanded towards the carboxy-terminal end of the docking domain in macroH2A (Fig. 4).

\section{The H2A L1 Loop May Select for the Second H2A-H2B Dimer}

The L1 loops of the two H2A moieties within the nucleosome are involved in the formation of the L1L1 interface, which is the only site of interaction between the two H2A-H2B dimers in the nucleosome. The L1L1 interface is responsible for the cooperative incorporation of the two H2A-H2B dimers. It may also stabilize the two gyres of the nucleosome core particle (Fig. 2D). Altering the biochemical nature of this interface should therefore result in an altered response to the transcriptional machinery and to chromatin remodeling factors, as well as determine the histone composition of the nucleosome.

There is no experimental evidence for the tacit assumption that one nucleosome contains, for example, two H2A.Z-H2B dimers. It is theoretically possible to have a nucleosome in which only one of the H2A moieties has been replaced by its corresponding variant, resulting in a nucleosome with one $(\mathrm{H} 3-\mathrm{H} 4)_{2}$ tetramer, one major H2A-H2B dimer, and one variant H2A-H2B dimer. From 
A

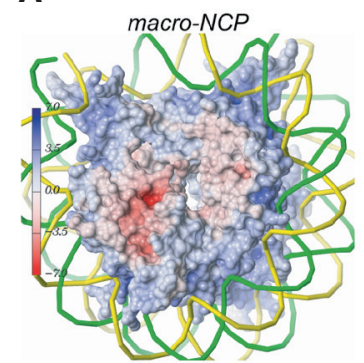

B

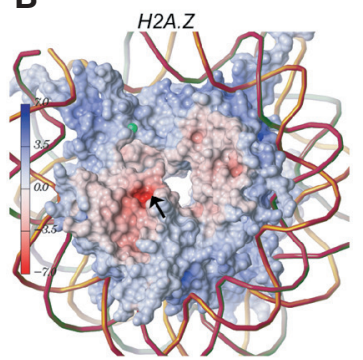

C

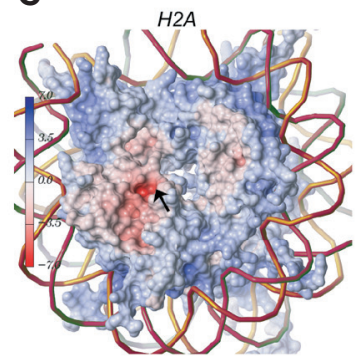

Figure 4. Subtle changes in the molecular surface of macro-NCP compared to H2A.Z-NCP and major-NCP. $(A)$ macro-NCP; $(B)$ H2A.Z-NCP; (C) major-NCP. The electrostatic potential ranges from +7.0 (blue) to -7.0 (red) kcal/mole/e (color bar); shown is the value at the solvent-accessible surface (out $1.4 \AA$, the radius of a water molecule, from the molecular surface) mapped back on to the molecular surface. Molecular surfaces were calculated with the program MSMS (Sanner et al. 1996) using a 1.4- $\AA$ probe sphere. The acidic patch that provides an essential crystal contact with the amino-terminal tail of histone $\mathrm{H} 4$ of a neighboring nucleosome core particle is indicated by a black arrow. (B,C, Reprinted, with permission, from Suto et al. 2000 [@Nature Publishing Group; http://www.nature.com/].)

a nucleosomal viewpoint, the primary determinant of the composition of a given nucleosome must be the compatibility of the L1 loop of major H2A with those of different $\mathrm{H} 2 \mathrm{~A}$ variants. To investigate this possibility in vitro, we performed salt-gradient reconstitutions with mixtures of $(\mathrm{H} 3-\mathrm{H} 4)_{2}$ tetramer, (H2A-H2B) dimers, and H2A.ZH2B dimers (Fig. 5); or with H2A.Bbd-H2B dimers (Fig. 6). We used either nickel-affinity chromatography to isolate nucleosomes containing his-tagged H2A (Fig. 5), or gel elution of "mixed nucleosome bands" (Fig. 6), followed by analysis of the histone content by SDS PAGE. Using these two approaches, we could show that while all histone H2A variants are capable of forming hybrid nucleosomes (Figs. 5 and 6 ), the propensity to do so clearly differs between macroH2A, H2A.Z, and H2A.Bbd.

Intriguingly, hybrid nucleosomes reconstituted from a mixture of H2A-H2B dimers and H2A.Bbd-H2B dimers together with $(\mathrm{H} 3-\mathrm{H} 4)_{2}$ tetramer protect only $\sim 130 \mathrm{bp}$ of DNA against digestion with micrococcal nuclease, that is, $\sim 12$ base pairs more than nucleosomes containing two H2A.Bbd chains and $\sim 15$ base pairs less than canonical nucleosome (not shown). The ability of H2A.Bbd to form hybrid nucleosomes is independent of the assembly pathway, since the same end result is obtained by yNAP-1-dependent assembly under physiological ionic strength (Fig. 6C).

Our results clearly indicate the possibility that variants may be combined with major-type histone $\mathrm{H} 2 \mathrm{~A}$ in a single nucleosome, thus generating yet another level of structural and functional heterogeneity. The specific nature of the different L1L1 interfaces and their potential influence on the accessibility of nucleosomal DNA will become more obvious in light of future structural studies. How would such nucleosomes be assembled in vivo? Histone H2A-H2B dimers are in rapid exchange even in the absence of transcription and replication (Louters and Chalkley 1985; Kimura and Cook 2001). Recently discovered histone-variant specific assembly factors for H2A.Z (Krogan et al. 2003; Kobor et al. 2004; Mizuguchi et al. 2004) promote the replacement of one or both H2AH2B dimer with a H2A.Z-H2B dimer. ATP-dependent chromatin remodeling factors are also capable of actively exchanging histone variant dimers into folded nucleosomes (Bruno et al. 2003). Since it is unlikely that both dimers are exchanged at the same time, the final "equilibrium" makeup of a particular nucleosome will be determined by the L1 loop, and by the relative availability of major-type and variant histones.

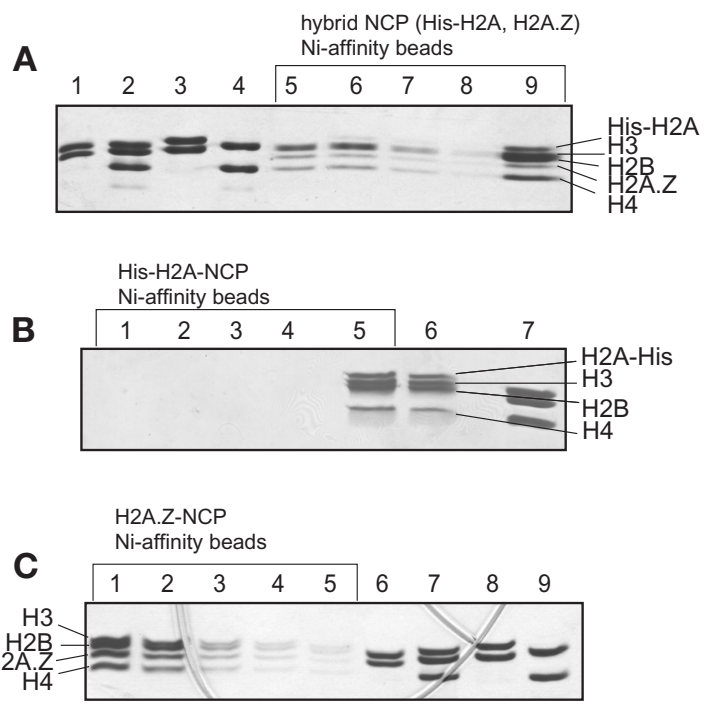

Figure 5. H2A.Z can form hybrid nucleosomes with major-type H2A. (A) Lane 1: H2A.Z-H2B dimer. Lane 2: Histone octamer with untagged $\mathrm{H} 2 \mathrm{~A}$. Lane 3: His-tagged $\mathrm{H} 2 \mathrm{~A}-\mathrm{H} 2 \mathrm{~B}$ dimer. Lane 4: H3-H4 tetramer. Lanes 5-8: Flowthrough and washes with 5mM imidazole of NCP reconstituted from a mixture of H2A.Z, His-tagged H2A, H2B, H3, and H4 after a 2-hr incubation with Ni-NTA beads. Lane 9: Elution with $1 \mathrm{M}$ imidazole. $(B)$ Control with his-tagged H2A NCP. Lanes 1-4: Flowthrough and washes with 5-mM imidazole after a 2-hr incubation of His-tagged H2A NCP to Ni-NTA beads. Lane 5: Elution with $1 \mathrm{M}$ imidazole. Lane 6: Onput. Lane 7: Histone octamer with untagged H2A. (C) Control with untagged H2A.Z nucleosomes. Lanes 1-4: Flowthrough and washes with 5-mm imidazole after 2-hr binding of H2A.Z NCPs to Ni-NTA beads. Lane 5: Elution with $1 \mathrm{M}$ imidazole. Lane 6: H2A.Z-H2B dimer. Lane 7: Histone octamer with non His-tagged H2A. Lane 8: His-tagged H2A-H2B dimer (His-tagged H2A, upper band; H2B, lower band). Lane 9: H3H4 tetramer. All gels shown here are 18\% SDS-PAGE gels stained with Coomassie brilliant blue. 
A

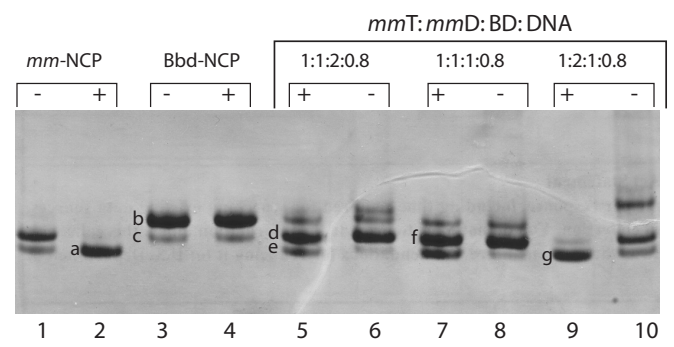

B

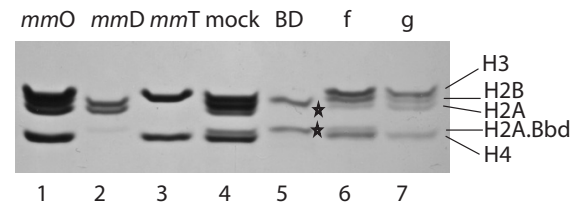

C

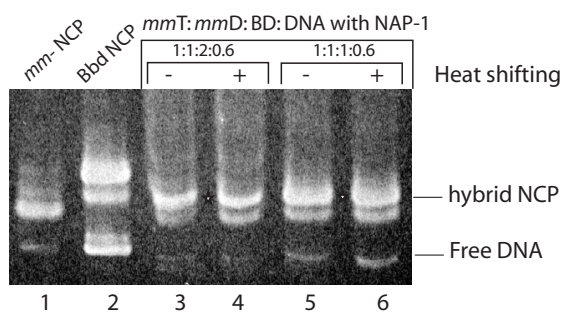

Figure 6. H2A.Bbd can form hybrid nucleosomes with majortype H2A. (A) Salt gradient reconstituted mouse NCP $(\mathrm{mm}-$ NCP; lanes 1 and 2), Bbd-NCP (lanes 3 and 4), and hybrid NCPs (lanes 5-10), before (-) and after $(+)$ a $1-$ hr incubation at $37^{\circ} \mathrm{C}$, were analyzed by $5 \%$ native gel and stained by Coomassie brilliant blue. Ratios between mouse $(\mathrm{H} 3-\mathrm{H} 4)_{2}$ tetramer $(\mathrm{mm} \mathrm{T})$, mouse $(\mathrm{H} 2 \mathrm{~A}-\mathrm{H} 2 \mathrm{~B})$ dimer $(m m \mathrm{D})$, H2A.Bbd-H2B dimer (BD), and DNA for "hybrid NCP" reconstitution are indicated. Bands that were subsequently excised from the gel for further analysis are labeled $a-g$. (B) Bands $f, g$ (from $A$ ) were analyzed by $18 \%$ SDS-PAGE, stained with Coomassie brilliant blue. Mouse H2A and $\mathrm{H} 2 \mathrm{~A} . \mathrm{Bbd}$ are indicated by stars. Mouse octamer (MO, lane 1), $\mathrm{MD}$ (lane 2), $m m \mathrm{~T}$ (lane 3), $m m \mathrm{~T}: m m \mathrm{D}: \mathrm{BD}$ mixtures of 1:1:1 (mock, lane 4), and BD (lane 5) are shown as controls. (C) Hybrid NCPs are also obtained with yNAP-1-dependent nucleosome assemblies. yNAP-1-reconstituted NCP (reconstituted at indicated ratios) was compared with NCP reconstituted over a salt gradient on a $5 \%$ native gel. The gel was stained with ethidium bromide. Asterisks: hybrid NCP.

\section{CONCLUSIONS AND OUTLOOK}

Histone variants are receiving an ever increasing amount of attention, but much needs to be done before we reach a complete understanding of their role in the complex biology of chromatin. In vitro, structural and biophysical analysis of nucleosomes containing H2A.Bbd and CenpA (in addition to the already available structures for H2A.Z- and macroH2A-containing nucleosomes) will provide further insight into the ways in which nucleosome structure and dynamics is affected by histone variants. These studies will also have to be expanded to take into the account the possible existence of hybrid nucleosomes. Second, we will need to extend our investigations to include model nucleosomal arrays, as demonstrated for H2A.Z (Fan et al. 2002). Third, the interaction of variantcontaining chromatin with various linker histones and nonhistone proteins needs to be investigated. Fourth, more systematic analyses are needed to study the dy- namic behavior of variant-containing nucleosomes and chromatin in the presence of various chromatin remodeling factors (see, e.g., Flaus et al. 2004). Finally, the transcriptional properties of variant-containing nucleosomes from several model promoters will have to be scrutinized carefully in vitro.

In vivo, the challenges are perhaps even greater. For example, knockout models are available for only a few histone variants. Second, we need to investigate the distribution of histone variants at the single-nucleosome (and subnucleosome) level at a variety of chromatin loci. This represents a technical challenge that may not be easily overcome. Third, the pathways by which variant nucleosomes (or hybrid nucleosomes) are assembled need to be further investigated. Finally, the ability of histone variants to be posttranslationally modified must be scrutinized. Combined, these studies are likely to yield a picture of mind-boggling complexity that probably will approach or even exceed that of the histone modification network. Doubtlessly, histone variants add an entirely new dimension to the "histone code."

\section{REFERENCES}

Ahmad K. and Henikoff S. 2002a. Histone H3 variants specify modes of chromatin assembly. Proc. Natl. Acad. Sci. (suppl. 4) 99: 16477 .

. 2002b. The histone variant H3.3 marks active chromatin by replication-independent nucleosome assembly. Mol. Cell 9: 1191 .

Angelov D., Molla A., Perche P.Y., Hans F., Cote J., Khochbin S., Bouvet P., and Dimitrov S. 2003. The histone variant macroH2A interferes with transcription factor binding and SWI/SNF nucleosome remodeling. Mol. Cell 11: 1033.

Bao Y., Konesky K., Park Y.J., Rosu S., Dyer P.N., Rangasamy D., Tremethick D.J., Laybourn P.J., and Luger K. 2004. Nucleosomes containing the histone variant H2A.Bbd organize only 118 base pairs of DNA. EMBO J. 23: 3314 .

Bassing C.H., Suh H., Ferguson D.O., Chua K.F., Manis J., Eckersdorff M., Gleason M., Bronson R., Lee C., and Alt F.W. 2003. Histone H2AX: A dosage-dependent suppressor of oncogenic translocations and tumors. Cell 114: 359.

Black E.B., Foltz D.R., Chakravarthy S., Luger K., Woods V.L.J., and Cleveland D.W. 2004. Structural determinants for generating centromeric chromatin. Nature 430: 578.

Bruno M., Flaus A., Stockdale C., Rencurel C., Ferreira H., and Owen-Hughes T. 2003. Histone H2A/H2B dimer exchange by ATP-dependent chromatin remodeling activities. Mol. Cell 12: 1599 .

Celeste A., Fernandez-Capetillo O., Kruhlak M.J., Pilch D.R., Staudt D.W., Lee A., Bonner R.F., Bonner W.M., and Nussenzweig A. 2003. Histone H2AX phosphorylation is dispensable for the initial recognition of DNA breaks. Nat. Cell Biol. 5: 675.

Chadwick B.P. and Willard H.F. 2001. A novel chromatin protein, distantly related to histone $\mathrm{H} 2 \mathrm{~A}$, is largely excluded from the inactive X chromosome. J. Cell Biol. 152: 375.

Chadwick B.P., Valley C.M., and Willard H.F. 2001. Histone variant macroH2A contains two distinct macrochromatin domains capable of directing macroH2A to the inactive $\mathrm{X}$ chromosome. Nucleic Acids Res. 29: 2699.

Chen T.A., Smith M.M., Le S.Y., Sternglanz R., and Allfrey V.G. 1991. Nucleosome fractionation by mercury affinity chromatography. Contrasting distribution of transcriptionally active DNA sequences and acetylated histones in nucleosome fractions of wild-type yeast cells and cells expressing a histone $\mathrm{H} 3$ gene altered to encode a cysteine 110 residue. J. Biol. Chem. 266: 6489. 
Clarkson M.J., Wells J.R., Gibson F., Saint R., and Tremethick D.J. 1999. Regions of variant histone His2AvD required for Drosophila development. Nature 399: 694.

Costanzi C. and Pehrson J.R. 1998. Histone macroH2A1 is concentrated in the inactive $\mathrm{X}$ chromosome of female mammals. Nature 393: 599.

2001. MacroH2A2, a new member of the MacroH2A core histone family. J. Biol. Chem. 276: 21776.

Crane-Robinson C. 1997. Where is the globular domain of linker histone located on the nucleosome? Trends Biochem. Sci. 22: 75.

Faast R., Thonglairoam V., Schulz T.C., Beall J., Wells J.R., Taylor H., Matthaei K., Rathjen P.D., Tremethick D.J., and Lyons I. 2001. Histone variant H2A.Z is required for early mammalian development. Curr. Biol. 11: 1183.

Fan J.Y., Gordon F., Luger K., Hansen J.C., and Tremethick D.J. 2002. The essential histone variant H2A.Z regulates the equilibrium between different chromatin conformational states. Nat. Struct. Biol. 19: 172.

Flaus A., Rencurel C., Ferreira H., Wiechens N., and OwenHughes T. 2004. Sin mutations alter inherent nucleosome mobility. EMBO J. 23: 343.

Gautier T., Abbott D.W., Molla A., Verdel A., Ausio J., and Dimitrov S. 2004. Histone variant H2ABbd confers lower stability to the nucleosome. EMBO Rep. 5: 715.

Georges S.A., Kraus W.L., Luger K., Nyborg J.K., and Laybourn P.J. 2002. p300-mediated tax transactivation from recombinant chromatin: Histone tail deletion mimics coactivator function. Mol. Cell. Biol. 22: 127.

Hamiche A., Carot V., Alilat M., De Lucia F., O’Donohue M.F., Revet B., and Prunell A. 1996. Interaction of the histone (H3H4)2 tetramer of the nucleosome with positively supercoiled DNA minicircles: Potential flipping of the protein from a leftto a right-handed superhelical form. Proc. Natl. Acad. Sci. 93: 7588.

Hansen J.C. 2002. Conformational dynamics of the chromatin fiber in solution: Determinants, mechanisms, and functions. Annu. Rev. Biophys. Biomol. Struct. 31: 361.

Hayes J.J. and Hansen J.C. 2001. Nucleosomes and the chromatin fiber. Curr. Opin. Genet. Dev. 11: 124.

Henikoff S., Furuyama T., and Ahmad K. 2004. Histone variants, nucleosome assembly and epigenetic inheritance. Trends Genet. 20: 320.

Hoyer-Fender S., Costanzi C., and Pehrson J.R. 2000. Histone macroH2A 1.2 is concentrated in the XY-body by the early pachytene stage of spermatogenesis. Exp. Cell Res. 258: 254.

Kimura H. and Cook P.R. 2001. Kinetics of core histones in living human cells: Little exchange of $\mathrm{H} 3$ and $\mathrm{H} 4$ and some rapid exchange of H2B. J. Cell Biol. 153: 1341.

Kobor M.S., Venkatasubrahmanyam S., Meneghini M.D., Gin J.W., Jennings J.L., Link A.J., Madhani H.D., and Rine J. 2004. A protein complex containing the conserved Swi2/Snf2-related ATPase Swr1p deposits histone variant H2A.Z into euchromatin. PLoS Biol. 2: E131.

Krogan N.J., Keogh M.C., Datta N., Sawa C., Ryan O.W., Ding H., Haw R.A., Pootoolal J., Tong A., Canadien V., Richards D.P., Wu X., Emili A., Hughes T.R., Buratowski S., and Greenblatt J.F. 2003. A Snf2 family ATPase complex required for recruitment of the histone H2A variant Htz1. Mol. Cell 12: 1565.

Liu X., Li B., and Gorovsky M.A. 1996. Essential and nonessential histone H2A variants in Tetrahymena thermophila. Mol. Cell. Biol. 16: 4305.

Louters L. and Chalkley R. 1985. Exchange of histones H1, H2A, and H2B in vivo. Biochemistry 24: 3080.

Luger K. and Richmond T.J. 1998a. DNA binding within the nucleosome core. Curr. Opin. Struct. Biol. 8: 33.

. 1998b. The histone tails of the nucleosome. Curr. Opin. Genet. Dev. 8: 140.

Luger K., Maeder A.W., Richmond R.K., Sargent D.F., and Richmond T.J. 1997. X-ray structure of the nucleosome core particle at $2.8 \AA$ A resolution. Nature 389: 251.
Malik H.S. and Henikoff S. 2003. Phylogenomics of the nucleosome. Nat. Struct. Biol. 10: 882.

Meneghini M.D., Wu M., and Madhani H.D. 2003. Conserved histone variant H2A.Z protects euchromatin from the ectopic spread of silent heterochromatin. Cell 112: 725.

Mizuguchi G., Shen X., Landry J., Wu W.H., Sen S., and Wu C. 2004. ATP-driven exchange of histone H2AZ variant catalyzed by SWR1 chromatin remodeling complex. Science 303: 343 .

Palmer D.K., O’Day K., Trong H.L., Charbonneau H., and Margolis R.L. 1991. Purification of the centromere-specific protein CENP-A and demonstration that it is a distinctive histone. Proc. Natl. Acad. Sci. 88: 3734.

Park Y.J., Dyer P.N., Tremethick D.J., and Luger K. 2004. A new fluorescence resonance energy transfer approach demonstrates that the histone variant $\mathrm{H} 2 \mathrm{AZ}$ stabilizes the histone octamer within the nucleosome. J. Biol. Chem. 279: 24274.

Pehrson J.R. and Fried V.A. 1992. MacroH2A, a core histone containing a large nonhistone region. Science 257: 1398.

Pehrson J.R., Costanzi C., and Dharia C. 1997. Developmental and tissue expression patterns of histone macroH2A1 subtypes. J. Cell. Biochem. 65: 107.

Perche P.Y., Vourc'h C., Konecny L., Souchier C., RobertNicoud M., Dimitrov S., and Khochbin S. 2000. Higher concentrations of histone macroH2A in the Barr body are correlated with higher nucleosome density. Curr. Biol. 10: 1531.

Protacio R.U. and Widom J. 1996. Nucleosome transcription studied in a real-time synchronous system: Test of the lexosome model and direct measurement of effects due to histone octamer. J. Mol. Biol. 256: 458.

Rangasamy D., Greaves I., and Tremethick D.J. 2004. RNA interference demonstrates a novel role for H2A.Z in chromosome segregation. Nat. Struct. Mol. Biol. 11: 650.

Rangasamy D., Berven L., Ridgway P., and Tremethick D.J. 2003. Pericentric heterochromatin becomes enriched with H2A.Z during early mammalian development. EMBO J. 22: 1599.

Rasmussen T.P., Huang T., Mastrangelo M.A., Loring J., Panning B., and Jaenisch R. 1999. Messenger RNAs encoding mouse histone macroH2A1 isoforms are expressed at similar levels in male and female cells and result from alternative splicing. Nucleic Acids Res. 27: 3685.

Richmond T.J. and Davey C.A. 2003. The structure of DNA in the nucleosome core. Nature 423: 145.

Rothkamm K. and Lobrich M. 2003. Evidence for a lack of DNA double-strand break repair in human cells exposed to very low x-ray doses. Proc. Natl. Acad. Sci. 100: 5057.

Sanner M.F., Olson A.J., and Spehner J.C. 1996. Reduced surface: An efficient way to compute molecular surfaces. Biopolymers 38: 305 .

Shelby R.D., Vafa O., and Sullivan K.F. 1997. Assembly of CENP-A into centromeric chromatin requires a cooperative array of nucleosomal DNA contact sites. J. Cell Biol. 136: 501 .

Sullivan K.F., Hechenberger M., and Masri K. 1994. Human CENP-A contains a histone $\mathrm{H} 3$ related histone fold domain that is required for targeting to the centromere. J. Cell Biol. 127: 581 .

Sullivan S., Sink D.W., Trout K.L., Makalowska I., Taylor P.M., Baxevanis A.D., and Landsman D. 2002. The histone database. Nucleic Acids Res. 30: 341.

Suto R.K., Clarkson M.J., Tremethick D.J., and Luger K. 2000. Crystal structure of a nucleosome core particle containing the variant histone H2A.Z. Nat. Struct. Biol. 7: 1121.

Suto R.K., Edayathumangalam R.S., White C.L., Melander C., Gottesfeld J.M., Dervan P.B., and Luger K. 2003. Crystal structures of nucleosome core particles in complex with minor groove DNA-binding ligands. J. Mol. Biol. 326: 371.

van Daal A. and Elgin S.C. 1992. A histone variant, H2AvD, is essential in Drosophila melanogaster. Mol. Biol. Cell 3: 593.

Vermaak D. and Wolffe A.P. 1998. Chromatin and chromosomal controls in development. Dev. Genet. 22: 1. 


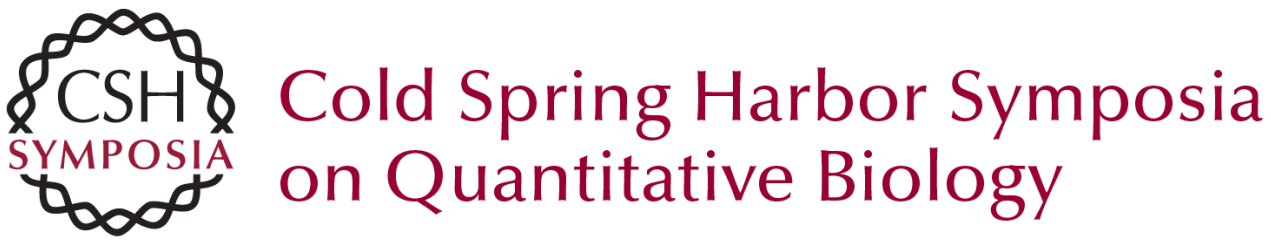

\section{Structural Characterization of Histone H2A Variants}

S. CHAKRAVARTHY, Y. BAO, V.A. ROBERTS, et al.

Cold Spring Harb Symp Quant Biol 2004 69: 227-234

Access the most recent version at doi:10.1101/sqb.2004.69.227

References This article cites 55 articles, 19 of which can be accessed free at: http://symposium.cshlp.org/content/69/227.full.html\#ref-list-1

\section{License}

Email Alerting Receive free email alerts when new articles cite this article - sign up in Service the box at the top right corner of the article or click here. 